\title{
Approximations for Soft Fuzzy Rough Sets
}

\author{
I. Beg, T. Rashid
}

\begin{abstract}
In this paper, we introduce a modified soft fuzzy rough set model. The lower and upper approximation operators are presented and their related properties are investigated. It is shown that these new models of approximations are finer than already known in the literature

Keywords: Fuzzy set; rough set; soft set; soft fuzzy rough set; approximations.
\end{abstract}

\section{Introduction}

Several notions have been presented in the literature to cope with the uncertainty, vagueness and ambiguity, like fuzzy set theory [23], rough set theory [19] and soft set theory [18]. Each of these ideas have its inherent difficulties as pointed out in [18]. Fuzzy set theory has been used to handle imprecision in decision making problems to take care of the ambiguity in an information $[2,3]$. Systematic treatment of deductive aspects and structures of fuzzy logic are presented in [8]. Applications of rough sets in various fields can also be seen in $[7,10,11]$. Molodtsov [18] introduced the concept of soft set theory as a new mathematical tool to deal with uncertainty. Now a days development on soft set theory is progressing rapidly. Maji et al. [16] defined several operations on soft sets and gave detailed study on the theory of soft sets. Afterward Ali et al. [1] proved that De Morgan's laws hold in soft set theory. Lattice structure is developed for soft set theory by Qin and Hong [20] and they defined the notion of soft quotient algebras of soft sets. Maji et al. [15] proposed a decision making method based on soft set and rough sets. Chen et al. [4] presented soft set parameterization reduction, and compared this notion to the related concept of attributes reduction in rough set theory. Kong et al. [13] defined the notion of normal parameter reduction of soft sets and developed a reduction algorithm based on the importance degree of parameters. Maji et al. [14] introduced the notion of fuzzy soft sets by combining fuzzy sets and soft sets because in many cases parameters in soft sets are vague. Roy and Maji

Manuscript received March 29, 2016; accepted July 17, 2016.

Ismat Beg is with the Lahore School of Economics, Lahore, Pakistan; Tabasam Rashid is with the University of Management and Technology, Lahore, Pakistan. 
[21] developed a fuzzy soft set theoretic approach towards a decision making problem. Xiao et al. [22] proposed a combined forecasting approach based on fuzzy soft set theory. This theory is now widely applied in many real world problems and in the development of new mathematical structures [6, 9, 12]. Recently Meng et al. [17] proposed the concept of soft fuzzy rough set and developed some important operators for the approximations of soft fuzzy rough sets. In these approximation operators there are some shortcomings (undefinable set will not always have upper or lower approximation). The purpose of this paper is to overcome these shortcomings by improving the basic structure of soft fuzzy rough set. Rest of this paper is arranged in the following manner. In Section 2, some basic notions are given to understand our proposal. In Section 3, modified soft fuzzy rough sets and its approximation operators are developed. In Section 4, conclusion of the paper is given.

\section{Preliminaries}

First we review some basic concepts, necessary to understand our proposal.

Let $U$ be a crisp universe of generic elements, a fuzzy set $\mathscr{A}$ in the universe $U$ is a mapping from $U$ to $[0,1]$. For any $u \in U$, the value $\mathscr{A}(u)$ is called the degree of membership of $u$ in $\mathscr{A}$. If membership value of the elements is 0 or 1 then that fuzzy set is also called as crisp set. So the membership value of all the elements in universal set $U$ is 1 and the membership value of all the elements in empty set is 0 . Universal set $U$ in the form of fuzzy set is $\mathscr{U}(u)=1$ for all $u \in U$. Similarly, empty set $\emptyset$ in the form of fuzzy set is $\emptyset(u)=0$ for all $u \in U$. The family of all subsets of $U$ is denoted by $P(U)$ and family of all fuzzy sets in $U$ is denoted by $F S(U)$. With the min-max system proposed by Zadeh, fuzzy set intersection, union and complement are defined component wise as follow:

$$
\begin{aligned}
& (\mathscr{A} \cap \mathscr{B})(u)=\mathscr{A}(u) \wedge \mathscr{B}(u), \\
& (\mathscr{A} \cup \mathscr{B})(u)=\mathscr{A}(u) \vee \mathscr{B}(u), \\
& \mathscr{A}^{c}(u)=1-\mathscr{A}(u),
\end{aligned}
$$

where $\mathscr{A}, \mathscr{B}$ are fuzzy sets and $u \in U$. By $\mathscr{A} \subseteq \mathscr{B}$, we mean that $\mathscr{A}(u) \leq \mathscr{B}(u)$ for all $u \in U$. Clearly, $\mathscr{A}=\mathscr{B}$ if $\mathscr{A}(u)=\mathscr{B}(u)$ for all $u \in U$.

Definition 1 [24] $\alpha$-level set of $\mathscr{A}$ is defined as $(\mathscr{A})_{\alpha}=\{u \in U ; \mathscr{A}(u)>\alpha\}$.

In 1999, Molodtsov [18] introduced the concept of soft sets. Let $U$ be the universe set and $E$ the set of all possible parameters under consideration with respect to $U$. Usually, parameters are attributes, characteristics, or properties of objects in $U$. Molodtsov [18] defined the notion of a soft set in the following way:

Definition 2 [18] A pair $(F, A)$ is called a soft set over $U$, where $A \subseteq E$ and $F$ is a mapping given by $F: A \rightarrow P(U)$. In other words, a soft set over $U$ is a parameterized family of 
subsets of $U$. For $e \in A, F(e)$ may be considered as the set of e-approximate elements of the soft set $(F, A)$.For $u \in U, F(e) u=1$ if $u \in F(e)$ and $F(e) u=0$ if $u \notin F(e)$.

Definition 3 [5] Let $S=(F, A)$ be a soft set over $U$. Then the pair $S A S=(U, S)$ is called a soft approximation space. Based on SAS, following two operations are defined:

$$
\begin{aligned}
& \underline{\operatorname{sra}}_{S A S}(X)=\{u \in U: \exists a \in A[u \in F(a) \subseteq X]\} \\
& \overline{\operatorname{sra}}_{S A S}(X)=\{u \in U: \exists a \in A[u \in F(a), F(a) \cap X \neq \emptyset]\}
\end{aligned}
$$

for any subset $X$ of $U$. Two subsets $\underline{s r a}_{S A S}(X)$ and $\overline{s r a}_{S A S}(X)$ called the lower and upper soft rough approximations of $X$ in $S A S$, respectively are obtained. If $\underline{\operatorname{sra}}_{S A S}(X)=\overline{\operatorname{sra}}_{S A S}(X)$, $X$ is said to be soft definable; otherwise $X$ is called a soft rough set.

Definition 4 [5] Let $S=(F, A)$ be a soft set over $U$. If $\bigcup_{a \in A} F(a)=U$, then $S$ is called a full soft set.

Definition 5 [16] Let $U$ be the universe set and $E$ the set of all possible parameters under consideration with respect to $U$. A pair $(\tilde{F}, A)$ is called a fuzzy soft set over $U$, where $A \subseteq E$ and $\tilde{F}$ is a mapping given by $\tilde{F}: A \rightarrow F S(U)$. For $e \in A$ and $u \in U, \tilde{F}(e) u$ is the membership value of $u$ in $\tilde{F}(e)$.

In the definition of a fuzzy soft set, fuzzy sets in the universe $U$ are used as substitutes for the crisp subsets of $U$. Hence, every soft set may be considered as a fuzzy soft set.

Definition 6 [17] Let $\tilde{S}=(\tilde{F}, A)$ be a fuzzy soft set over $U$, where $\tilde{F}$ is a map $\tilde{F}: A \rightarrow$ $F S(U)$. Then the pair $S F A S=(U, \tilde{S})$ is called soft fuzzy approximation space. For any fuzzy set $\mathscr{A} \in F S(U)$, the lower and upper soft fuzzy rough approximations of $\mathscr{A}$ with respect to $S F A S$ are denoted by $\underline{S F R A}_{S F A S}(\mathscr{A})$ and $\overline{S F R A}_{S F A S}(\mathscr{A})$, respectively, which are fuzzy sets in $U$ given by:

$$
\begin{aligned}
& \underline{S F R A}_{S F A S}(\mathscr{A})(x)=\bigwedge_{a \in A}\left((1-\tilde{F}(a)(x)) \vee\left(\bigwedge_{y \in U}((1-\tilde{F}(a)(y)) \vee \mathscr{A}(y))\right)\right) \\
& \overline{\operatorname{SFRA}}_{S F A S}(\mathscr{A})(x)=\bigvee_{a \in A}\left(\tilde{F}(a)(x)\left(\bigvee_{y \in U}(\tilde{F}(a)(y) \wedge \mathscr{A}(y))\right)\right)
\end{aligned}
$$

for all $x \in U$. The operators $\underline{S F R A}_{S F A S}$ and $\overline{S F R A}_{S F A S}$ are called the lower and upper soft fuzzy rough approximation operators on fuzzy sets. If $\underline{S F R A}_{S F A S}(\mathscr{A})=\overline{S F R A}_{S F A S}(\mathscr{A})$, $\mathscr{A}$ is said to be soft fuzzy definable; otherwise $\mathscr{A}$ is called a soft fuzzy rough set.

\section{Modified soft fuzzy rough set}

Meng et al. [17] showed that the approximations of soft fuzzy rough set are extensions of Feng's approximations of soft rough set. In continuation of these results here we propose 
the modified soft fuzzy rough set. This extension provided a better and finer approximations of soft fuzzy rough sets than Meng's approximations of these sets.

Definition 7 Let $(\tilde{F}, A)$ be a fuzzy soft set over $U$, where $\tilde{F}$ is a map $\tilde{F}: A \rightarrow F S(U)$. Let $\varphi: U \rightarrow F S(A)$ be another map defined as $\varphi(x)=\left\{\tilde{F}(a) x: x \in(\tilde{F}(a))_{0}\right\}$ where $\tilde{F}(a) x$ is the membership value of a in $\varphi(x)$. Then the pair MSFAS $=(U, \varphi)$ is called modified soft fuzzy approximation space. For any fuzzy set $\mathscr{A} \in F S(U)$, the lower and upper modified soft fuzzy rough approximations of $\mathscr{A}$ with respect to MSFAS are denoted by $\operatorname{MSFRA}_{M S F A S}(\mathscr{A})$ and $\overline{M S F R A}_{M S F A S}(\mathscr{A})$, respectively, which are fuzzy sets in $U$ given by:

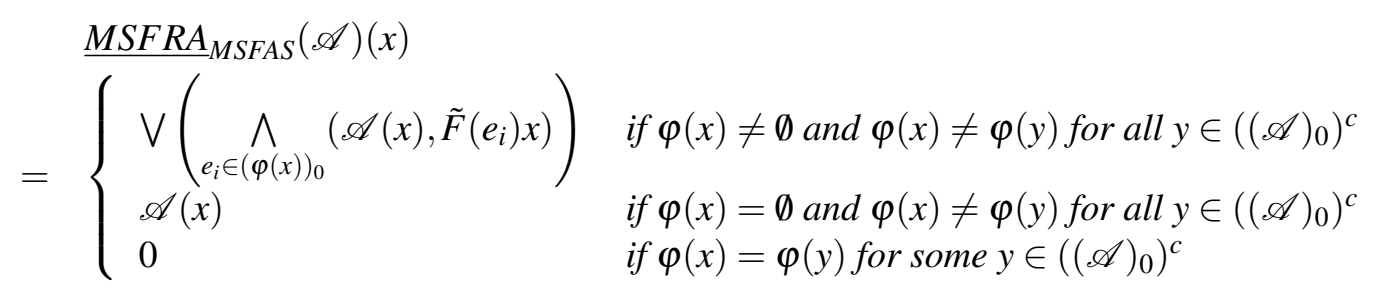

for all $x \in\left(\mu_{\mathscr{A}}\right)_{0}$ and

$$
\begin{aligned}
& \overline{\operatorname{MSFRA}}_{M S F A S}(\mathscr{A})(x) \\
= & \begin{cases}\wedge\left(\bigvee_{e_{i} \in(\varphi(x))_{0}}\left(\mathscr{A}(x), \tilde{F}\left(e_{i}\right) x\right)\right) & \text { if } \varphi(x) \neq \emptyset \text { and } \varphi(x)=\varphi(y) \text { for some } y \in(\mathscr{A})_{0} \\
\mathscr{A}(x) & \text { if } \varphi(x)=\emptyset \text { and } \varphi(x)=\varphi(y) \text { for some } y \in(\mathscr{A})_{0} \\
0 & \text { if } \varphi(x) \neq \varphi(y) \text { for all } y \in(\mathscr{A})_{0}\end{cases}
\end{aligned}
$$

for all $x \in U$.

The operators $\underline{M S F R A}_{M S F A S}$ and $\overline{M S F R A}_{M S F A S}$ are called the lower and upper modified soft fuzzy rough approximation operators on fuzzy sets. If $\underline{M S F R A}_{M S F A S}(\mathscr{A}) \neq \overline{M S F R A}_{M S F A S}(\mathscr{A})$, $\mathscr{A}$ is said to be a modified soft fuzzy rough set.

Remark 1 For any fuzzy set $\mathscr{A}$, it is easy to see that $\emptyset \subseteq \operatorname{MSFRA}_{M S F A S}(\mathscr{A}) \subseteq U$ and $\emptyset \subseteq \overline{M S F R A}_{M S F A S}(\mathscr{A}) \subseteq U$.

Theorem 1 Let $(\tilde{F}, A)$ be a fuzzy soft set over $U, M S F A S=(U, \varphi)$ be a modified soft approximation space and $\mathscr{A} \in F S(U)$. Then we have

1. $\underline{\operatorname{MSFRA}}_{M S F A S}(\mathscr{A}) \subseteq \mathscr{A} \subseteq \overline{\operatorname{MSFRA}}_{M S F A S}(\mathscr{A})$,

2. $\underline{\operatorname{MSFRA}}_{M S F A S}(\mathscr{U}) \subseteq \mathscr{U}=\overline{M S F R A}_{M S F A S}(\mathscr{U})$,

3. $\underline{\operatorname{MSFRA}}_{M S F A S}(\emptyset)=\emptyset=\overline{\operatorname{MSFRA}}_{M S F A S}(\emptyset)$.

Proof We use the standard pointwise argument. 
1. There are three cases for $\underline{M S F R A}_{M S F A S}(\mathscr{A})(x)$.

Case i. If $\underline{\operatorname{MSFRA}}_{M S F A S}(\mathscr{A})(x)=\mathscr{A}(x)$ then we can write that

$$
\underline{\operatorname{MSFRA}}_{M S F A S}(\mathscr{A})(x) \leq \mathscr{A}(x) .
$$

Case ii. If $\underline{\operatorname{MSFRA}}_{M S F A S}(\mathscr{A})(x)=0$ then $\underline{\operatorname{MSFRA}}_{M S F A S}(\mathscr{A})(x) \leq \mathscr{A}(x)$ because we know that $0 \leq \mathscr{A}(x) \leq 1$.

Thus, $\underline{M S F R A}_{M S F A S}(\mathscr{A})(x) \leq \mathscr{A}(x)$.

Case iii. If $\underline{\operatorname{MSFRA}}_{M S F A S}(\mathscr{A})(x)=\bigvee\left(\bigwedge_{e_{i} \in(\varphi(x))_{0}}\left(\mathscr{A}(x), \tilde{F}\left(e_{i}\right) x\right)\right)$ then it can be easily noted that $\left(\mathscr{A}(x) \wedge \tilde{F}\left(e_{i}\right) x\right) \leq \mathscr{A}(x)$ for all $e_{i} \in(\varphi(x))_{0}$.

This further implies that $\bigvee\left(\bigwedge_{e_{i} \in(\varphi(x))_{0}}\left(\mathscr{A}(x), \tilde{F}\left(e_{i}\right) x\right)\right) \leq \mathscr{A}(x)$.

Thus, $\underline{\operatorname{MSFRA}}_{M S F A S}(\mathscr{A})(x) \leq \mathscr{A}(x)$.

Now we want to prove that $\mathscr{A}(x) \leq \overline{M S F R A}_{M S F A S}(\mathscr{A})(x)$.

There are three cases for $\overline{M S F R A}_{M S F A S}(\mathscr{A})(x)$.

Case i. If $\overline{\operatorname{MSFRA}}_{M S F A S}(\mathscr{A})(x)=\wedge\left(\bigvee_{e_{i} \in(\varphi(x))_{0}}\left(\mathscr{A}(x), \tilde{F}\left(e_{i}\right) x\right)\right)$ then it can be noted that $\mathscr{A}(x) \leq\left(\mathscr{A}(x) \bigvee \tilde{F}\left(e_{i}\right) x\right)$ for all $e_{i} \in(\varphi(x))_{0}$.

This further implies that $\mathscr{A}(x) \leq \wedge\left(\bigvee_{e_{i} \in(\varphi(x))_{0}}\left(\mathscr{A}(x), \tilde{F}\left(e_{i}\right) x\right)\right)$

Thus $\mathscr{A}(x) \leq \overline{M S F R A}_{M S F A S}(\mathscr{A})(x)$.

Case ii. If $\overline{M S F R A}_{M S F A S}(\mathscr{A})(x)=\mathscr{A}(x)$ then $\mathscr{A}(x) \leq \overline{\operatorname{MSFRA}}_{M S F A S}(\mathscr{A})(x)$.

Case iii. $\overline{\operatorname{MSFRA}}_{M S F A S}(\mathscr{A})(x)=0$ when $\varphi(x) \neq \varphi(y)$ for all $y \in\left(\mu_{\mathscr{A}}\right)_{0}$, which further implies that $x \notin\left(\mu_{\mathscr{A}}\right)_{0}$. So $\mathscr{A}(x)=0$.

Thus $\mathscr{A}(x) \leq \overline{\operatorname{MSFRA}}_{M S F A S}(\mathscr{A})(x)$.

Hence

$$
\underline{\operatorname{MSFRA}}_{M S F A S}(\mathscr{A}) \subseteq \mathscr{A} \subseteq \overline{\operatorname{MSFRA}}_{M S F A S}(\mathscr{A})
$$

2. By (1), we can write that $\mathscr{U} \subseteq \overline{M S F R A}_{M S F A S}(\mathscr{U})$.

By definition of $\overline{M S F R A}_{M S F A S}(\mathscr{A})$, we can write $\overline{M S F R A}_{M S F A S}(\mathscr{A}) \subseteq \mathscr{U}$ for any fuzzy set $\mathscr{A}$. So $\overline{\operatorname{MSFRA}}_{M S F A S}(\mathscr{U}) \subseteq \mathscr{U}$. 
Thus, $\mathscr{U}=\overline{M S F R A}_{M S F A S}(\mathscr{U})$.

Since $\mathscr{U}(x)=1$ for all $x \in U$. So $\varphi(x) \neq \varphi(y)$ for all $y \in\left((\mathscr{U})_{0}\right)^{c}$ then there are two possible cases.

Case i. If $\varphi(x)=\emptyset$ then $\underline{M S F R A}_{M S F A S}(\mathscr{U})(x)=\mathscr{U}(x)$.

Case ii. If $\varphi(x) \neq \emptyset$ then $\underline{\operatorname{MSFRA}}_{M S F A S}(\mathscr{U})(x)=\mathrm{V}\left(\bigwedge_{e_{i} \in(\varphi(x))_{0}}\left(\mathscr{U}(x), \tilde{F}\left(e_{i}\right) x\right)\right)$.

It can be noted that $\left(\mathscr{U}(x) \wedge \tilde{F}\left(e_{i}\right) x\right) \leq \mathscr{U}(x)$ for all $e_{i} \in(\varphi(x))_{0}$, which further implies that $\bigvee\left(\bigwedge_{e_{i} \in(\varphi(x))_{0}}\left(\mathscr{U}(x), \tilde{F}\left(e_{i}\right) x\right)\right) \leq \mathscr{U}(x)$.

Thus $\underline{\operatorname{MSFRA}}_{M S F A S}(\mathscr{U})(x) \leq \mathscr{U}(x)$.

Hence

$$
\underline{\operatorname{MSFRA}}_{M S F A S}(\mathscr{U}) \subseteq \mathscr{U}=\overline{\operatorname{MSFRA}}_{M S F A S}(\mathscr{U}) .
$$

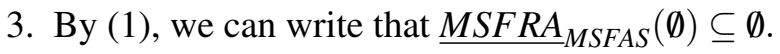

Since $\emptyset \subseteq \operatorname{MSFRA}_{M S F A S}(\mathscr{A})$ for any fuzzy set $\mathscr{A}$. So $\emptyset \subseteq \underline{M S F R A}_{M S F A S}(\emptyset)$.

Thus, $\underline{M S F R A}_{M S F A S}(\emptyset)=\emptyset$.

It is obvious that $(\emptyset)_{0}=\emptyset$.

So there is no $y$ in $(\emptyset)_{0}$.

This implies that $\varphi(x) \neq \varphi(y)$ for all $y \in\left(\mu_{\emptyset}\right)_{0}$. Thus, $\overline{\operatorname{MSFRA}}_{M S F A S}(\emptyset)(x)=0$ for all $x \in U$.

Hence

$$
\underline{\operatorname{MSFRA}}_{M S F A S}(\emptyset)=\emptyset=\overline{M S F R A}_{M S F A S}(\emptyset) .
$$

Theorem 2 Let $(\tilde{F}, A)$ be a fuzzy soft set over $U, M S F A S=(U, \varphi)$ be a modified soft fuzzy approximation space and $\mathscr{A}, \mathscr{B} \in F S(U)$. Then we have

1. $\mathscr{A} \subseteq \mathscr{B} \Rightarrow \underline{\operatorname{MSFRA}}_{M S F A S}(\mathscr{A}) \subseteq \underline{\operatorname{MSFRA}}_{M S F A S}(\mathscr{B})$,

2. $\mathscr{A} \subseteq \mathscr{B} \Rightarrow \overline{M S F R A}_{M S F A S}(\mathscr{A}) \subseteq \overline{M S F R A}_{M S F A S}(\mathscr{B})$.

Proof 
1. Let $\mathscr{A} \subseteq \mathscr{B}$ which implies that $\mathscr{A}(x) \leq \mathscr{B}(x)$ for all $x \in U$.

If $\underline{M S F R A}_{M S F A S}(\mathscr{A})(x)=0$ then it is easy to see that $\underline{\operatorname{MSFRA}}_{M S F A S}(\mathscr{A})(x) \leq \underline{\operatorname{MSFRA}}_{M S F A S}(\mathscr{B})(x)$ for all $x \in U$.

If $\varphi(x)=\emptyset$ and $\varphi(x) \neq \varphi(y)$ for all $y \in\left(\left(\mu_{\mathscr{A}}\right)_{0}\right)^{c}$ then $\underline{\operatorname{MSFRA}}_{M S F A S}(\mathscr{A})(x)=\mathscr{A}(x)$ where $x \in\left(\mu_{\mathscr{A}}\right)_{0}$. Since $\mathscr{A} \subseteq \mathscr{B}$ so $x \in\left(\mu_{\mathscr{B}}\right)_{0}$ then $\underline{\operatorname{MSFRA}}_{M S F A S}(\mathscr{B})(x)=\mathscr{B}(x)$.

Thus, $\underline{\operatorname{MSFRA}}_{M S F A S}(\mathscr{A})(x) \leq \underline{\operatorname{MSFRA}}_{M S F A S}(\mathscr{B})(x)$.

If $\varphi(x) \neq \emptyset$ and $\varphi(x) \neq \varphi(y)$ for all $y \in\left(\left(\mu_{\mathscr{A}}\right)_{0}\right)^{c}$ where $x \in\left(\mu_{\mathscr{A}}\right)_{0}$ then $x \in\left(\mu_{\mathscr{B}}\right)_{0}$ as $\mathscr{A} \subseteq \mathscr{B}$.

This implies that $\left(\mathscr{A}(x) \wedge \tilde{F}\left(e_{i}\right) x\right) \leq\left(\mathscr{B}(x) \wedge \tilde{F}\left(e_{i}\right) x\right)$ for all $e_{i} \in(\varphi(x))_{0}$, which further implies that $\bigvee\left(\bigwedge_{e_{i} \in(\varphi(x))_{0}}\left(\mathscr{A}(x), \tilde{F}\left(e_{i}\right) x\right)\right) \leq \bigvee\left(\bigwedge_{e_{i} \in(\varphi(x))_{0}}\left(\mathscr{B}(x), \tilde{F}\left(e_{i}\right) x\right)\right)$.

Thus, $\underline{\operatorname{MSFRA}}_{M S F A S}(\mathscr{A})(x) \leq \underline{\operatorname{MSFRA}}_{M S F A S}(\mathscr{B})(x)$.

Hence

$$
\underline{\operatorname{MSFRA}}_{M S F A S}(\mathscr{A}) \subseteq \underline{\operatorname{MSFRA}}_{M S F A S}(\mathscr{B}) .
$$

2. Let $\mathscr{A} \subseteq \mathscr{B}$ which implies that $\mathscr{A}(x) \leq \mathscr{B}(x)$ for all $x \in U$.

We want to show that $\overline{M S F R A}_{M S F A S}(\mathscr{A})(x) \leq \overline{M S F R A}_{M S F A S}(\mathscr{B})(x)$ for all $x \in U$.

If $\overline{\operatorname{MSFRA}}_{M S F A S}(\mathscr{B})(x)=1$ then $\overline{M S F R A}_{M S F A S}(\mathscr{A})(x) \leq \overline{\operatorname{MSFRA}}_{M S F A S}(\mathscr{B})(x)$.

Case i. If $\varphi(x) \neq \varphi(y)$ for all $y \in\left(\mu_{A}\right)_{0}$ then $\overline{M S F R A}_{M S F A S}(\mathscr{A})(x)=0$, which further implies that $\overline{M S F R A}_{M S F A S}(\mathscr{A})(x) \leq \overline{\operatorname{MSFRA}}_{M S F A S}(\mathscr{B})(x)$.

Case ii. If $\varphi(x)=\emptyset$ and $\varphi(x)=\varphi(y)$ for some $y \in\left(\mu_{A}\right)_{0}$ then the same $y \in\left(\mu_{B}\right)_{0}$ because of $\mathscr{A} \subseteq \mathscr{B}$. This further implies that $\overline{M S F R A}_{M S F A S}(\mathscr{A})(x)=\mathscr{A}(x)$ and $\overline{M S F R A}_{M S F A S}(\mathscr{B})(x)=\mathscr{B}(x)$.

Thus, $\overline{\operatorname{MSFRA}}_{M S F A S}(\mathscr{A})(x)=\overline{\operatorname{MSFRA}}_{M S F A S}(\mathscr{B})(x)$.

Case iii. If $\varphi(x) \neq \emptyset$ and $\varphi(x)=\varphi(y)$ for some $y \in\left(\mu_{A}\right)_{0}$ then the same $y \in\left(\mu_{B}\right)_{0}$ because of $\mathscr{A} \subseteq \mathscr{B}$.

This implies that $\left(\mathscr{A}(x) \bigvee \tilde{F}\left(e_{i}\right) x\right) \leq\left(\mathscr{B}(x) \bigvee \tilde{F}\left(e_{i}\right) x\right)$ for all $e_{i} \in(\varphi(x))_{0}$, which further implies that

$$
\bigwedge\left(\bigvee_{e_{i} \in(\varphi(x))_{0}}\left(\mathscr{A}(x), \tilde{F}\left(e_{i}\right) x\right)\right) \leq \bigwedge\left(\bigvee_{e_{i} \in(\varphi(x))_{0}}\left(\mathscr{B}(x), \tilde{F}\left(e_{i}\right) x\right)\right)
$$

Thus, $\overline{\operatorname{MSFRA}}_{M S F A S}(\mathscr{A})(x)=\overline{\operatorname{MSFRA}}_{M S F A S}(\mathscr{B})(x)$. 
Hence

$$
\overline{\operatorname{MSFRA}}_{M S F A S}(\mathscr{A}) \subseteq \overline{\operatorname{MSFRA}}_{M S F A S}(\mathscr{B})
$$

Theorem 3 Let $(\tilde{F}, A)$ be a fuzzy soft set over $U, M S F A S=(U, \varphi)$ be a modified soft fuzzy approximation space and $\mathscr{A}, \mathscr{B} \in F S(U)$. Then we have

1. $\overline{\operatorname{MSFRA}}_{M S F A S}(\mathscr{A} \cup \mathscr{B}) \supseteq \overline{\operatorname{MSFRA}}_{M S F A S}(\mathscr{A}) \cup \overline{\operatorname{MSFRA}}_{M S F A S}(\mathscr{B})$,

2. $\underline{\operatorname{MSFRA}}_{M S F A S}(\mathscr{A} \cap \mathscr{B}) \subseteq \underline{\operatorname{MSFRA}}_{M S F A S}(\mathscr{A}) \cap \underline{\operatorname{MSFRA}}_{M S F A S}(\mathscr{B})$,

3. $\underline{\operatorname{MSFRA}}_{M S F A S}(\mathscr{A} \cup \mathscr{B}) \supseteq \underline{\operatorname{MSFRA}}_{M S F A S}(\mathscr{A}) \cup \underline{M S F R A}_{M S F A S}(\mathscr{B})$,

4. $\overline{\operatorname{MSFRA}}_{M S F A S}(\mathscr{A} \cap \mathscr{B}) \subseteq \overline{M S F R A}_{M S F A S}(\mathscr{A}) \cap \overline{\operatorname{MSFRA}}_{M S F A S}(\mathscr{B})$.

Proof We use the standard pointwise argument.

1. Clearly $\mathscr{A} \cup \mathscr{B} \supseteq \mathscr{A}$ and $\mathscr{A} \cup \mathscr{B} \supseteq \mathscr{B}$. By using Theorem 2(2) we can say that $\overline{M S F R A}_{M S F A S}(\mathscr{A} \cup \mathscr{B}) \supseteq \overline{M S F R A}_{M S F A S}(\mathscr{A})$ and $\overline{M S F R A}_{M S F A S}(\mathscr{A} \cup \mathscr{B}) \supseteq \overline{M S F R A}_{M S F A S}(\mathscr{B})$.

Hence

$$
\overline{\operatorname{MSFRA}}_{M S F A S}(\mathscr{A} \cup \mathscr{B}) \supseteq \overline{\operatorname{MSFRA}}_{M S F A S}(\mathscr{A}) \cup \overline{M S F R A}_{M S F A S}(\mathscr{B}) .
$$

2. Clearly $\mathscr{A} \cap \mathscr{B} \subseteq \mathscr{A}$ and $\mathscr{A} \cap \mathscr{B} \subseteq \mathscr{B}$. By using Theorem 2(1) we can say that $\underline{M S F R A}_{M S F A S}(\mathscr{A} \cap \mathscr{B}) \subseteq \underline{\operatorname{MSFRA}}_{M S F A S}(\mathscr{A})$ and $\underline{\operatorname{MSFRA}}_{M S F A S}(\mathscr{A} \cap \mathscr{B}) \subseteq \underline{\operatorname{MSFRA}}_{M S F A S}(\mathscr{B})$.

Hence

$$
\underline{\operatorname{MSFRA}}_{M S F A S}(\mathscr{A} \cap \mathscr{B}) \subseteq \underline{\operatorname{MSFRA}}_{M S F A S}(\mathscr{A}) \cap \underline{\operatorname{MSFRA}}_{M S F A S}(\mathscr{B}) .
$$

3. Clearly $\mathscr{A} \cup \mathscr{B} \supseteq \mathscr{A}$ and $\mathscr{A} \cup \mathscr{B} \supseteq \mathscr{B}$. By using Theorem 2(1) we can say that ${\underline{M S F R A_{M S F A S}}}_{M}(\mathscr{A} \cup \mathscr{B}) \supseteq{\underline{M S F R A_{M S F A S}}}_{M}(\mathscr{A})$ and $\underline{\operatorname{MSFRA}}_{M S F A S}(\mathscr{A} \cup \mathscr{B}) \supseteq \underline{\operatorname{MSFRA}}_{M S F A S}(\mathscr{B})$.

Hence

$$
\underline{\operatorname{MSFRA}}_{M S F A S}(\mathscr{A} \cup \mathscr{B}) \supseteq \underline{\operatorname{MSFRA}}_{M S F A S}(\mathscr{A}) \cup \underline{\operatorname{MSFRA}}_{M S F A S}(\mathscr{B}) .
$$

4. Clearly $\mathscr{A} \cap \mathscr{B} \subseteq \mathscr{A}$ and $\mathscr{A} \cap \mathscr{B} \subseteq \mathscr{B}$. By using Theorem 2(2) we can say that $\overline{M S F R A}_{M S F A S}(\mathscr{A} \cap \mathscr{B}) \subseteq \overline{M S F R A}_{M S F A S}(\mathscr{A})$ and $\overline{M S F R A}_{M S F A S}(\mathscr{A} \cap \mathscr{B}) \subseteq \overline{M S F R A}_{M S F A S}(\mathscr{B})$. Hence

$$
\overline{\operatorname{MSFRA}}_{M S F A S}(\mathscr{A} \cap \mathscr{B}) \subseteq \overline{\operatorname{MSFRA}}_{M S F A S}(\mathscr{A}) \cap \overline{\operatorname{MSFRA}}_{M S F A S}(\mathscr{B})
$$


Theorem 4 Let $(\tilde{F}, A)$ be a fuzzy soft set over $U, M S F A S=(U, \varphi)$ be a modified soft fuzzy approximation space and $\mathscr{A} \in F S(U)$. Then we have

1. $\overline{\operatorname{MSFRA}}_{M S F A S}\left(\underline{\operatorname{MSFRA}}_{M S F A S}(\mathscr{A})\right) \supseteq \underline{\operatorname{MSFRA}}_{M S F A S}(\mathscr{A})$,

2. $\overline{\operatorname{MSFRA}}_{M S F A S}\left(\underline{\operatorname{MSFRA}}_{M S F A S}(\mathscr{A})\right) \supseteq \underline{\operatorname{MSFRA}}_{M S F A S}(\mathscr{A})$,

3. $\underline{\operatorname{MSFRA}}_{M S F A S}\left(\underline{\operatorname{MSFRA}}_{M S F A S}(\mathscr{A})\right) \subseteq \underline{\operatorname{MSFRA}}_{M S F A S}(\mathscr{A})$,

4. $\underline{\operatorname{MSFRA}}_{M S F A S}\left(\overline{\operatorname{MSFRA}}_{M S F A S}(\mathscr{A})\right) \subseteq \overline{\operatorname{MSFRA}}_{M S F A S}(\mathscr{A})$,

5. $\overline{\operatorname{MSFRA}}_{M S F A S}\left(\overline{\operatorname{MSFRA}}_{M S F A S}(\mathscr{A})\right) \supseteq \overline{\operatorname{MSFRA}}_{M S F A S}(\mathscr{A})$.

Proof We use the standard pointwise argument.

1. By Theorem 1(1) $\mathscr{A}(x) \leq \overline{M S F R A}_{M S F A S}(\mathscr{A})(x)$ for any fuzzy set $\mathscr{A}$. Now replace $\mathscr{A}$ by $\underline{\operatorname{MSFRA}}_{M S F A S}(\mathscr{A})$ then we get $\underline{\operatorname{MSFRA}}_{M S F A S}(\mathscr{A})(x) \leq \overline{\operatorname{MSFRA}}_{M S F A S}\left(\underline{\operatorname{MSFRA}}_{M S F A S}(\mathscr{A})\right)(x)$.

Hence

$$
\underline{\operatorname{MSFRA}}_{M S F A S}(\mathscr{A})(x) \subseteq \overline{\operatorname{MSFRA}}_{M S F A S}\left(\underline{\operatorname{MSFRA}}_{M S F A S}(\mathscr{A})\right)(x) .
$$

2. By Theorem 1(1) $\underline{M S F R A}_{M S F A S}\left(\mu_{\mathscr{A}}\right) \subseteq \mu_{\mathscr{A}}$ and by using Theorem 2(1) it can be written that $\underline{\operatorname{MSFRA}}_{M S F A S}\left(\underline{M S F R A}_{M S F A S}(\mathscr{A})\right) \subseteq \underline{\operatorname{MSFRA}} \underline{M S F A S}(\mathscr{A})$.

3. By Theorem 1(1) $\underline{M S F R A}_{M S F A S}(\mathscr{A})(x) \leq \mathscr{A}(x)$ for any fuzzy set $\mathscr{A}$. Now replace $\mathscr{A}$ by $\overline{M S F R A}_{M S F A S}(\mathscr{A})$ then we get

$$
\underline{\operatorname{MSFRA}}_{M S F A S}\left(\overline{\operatorname{MSFRA}}_{M S F A S}(\mathscr{A})\right)(x) \leq \overline{\operatorname{MSFRA}}_{M S F A S}(\mathscr{A})(x) .
$$

Hence

$$
\underline{\operatorname{MSFRA}}_{M S F A S}\left(\overline{\operatorname{MSFRA}}_{M S F A S}(\mathscr{A})\right) \subseteq \overline{\operatorname{MSFRA}}_{M S F A S}(\mathscr{A}) .
$$

4. By Theorem 1(1) $\mu_{\mathscr{A}} \subseteq \overline{M S F R A}_{M S F A S}(\mathscr{A})$ and by using Theorem 2(2) it can be written that $\overline{M S F R A}_{M S F A S}\left(\overline{M S F R A}_{M S F A S}(\mathscr{A})\right) \supseteq \overline{M S F R A}_{M S F A S}(\mathscr{A})$.

Remark 2 The fuzzification presented in this proposal uses Godel conjunction and disjunction (min and max) and Lukasiewicz negation. All the results remain true for all other type of the standard fuzzy connectives (e.g. product conjunction, disjunction and negation, Lukasiewicz conjunction and disjunction). 
Example 1 Let $U=\left\{u_{1}, u_{2}, u_{3}, u_{4}, u_{5}, u_{6}\right\}$ be the set of nine electricity provider operators (universe set) and $A=\left\{e_{1}, e_{2}, e_{3}, e_{4}\right\} \subseteq E$, where $e_{1}$ represents the online billing facility, $e_{2}$ represents fluctuation in voltage, $e_{3}$ represents life security breakers, $e_{4}$ represents after sale service. The soft fuzzy set $(\tilde{F}, A)$ is representing this data in Table 1.

$$
\begin{aligned}
& \tilde{F}: A \rightarrow P(U) \\
& \varphi: U \rightarrow P(A)
\end{aligned}
$$

Table 1. Soft set $(F, A)$

\begin{tabular}{l|llllll}
\hline & $u_{1}$ & $u_{2}$ & $u_{3}$ & $u_{4}$ & $u_{5}$ & $u_{6}$ \\
\hline$e_{1}$ & 0.2 & 0.8 & 0.8 & 0.2 & 0.8 & 0.2 \\
$e_{2}$ & 0.5 & 0 & 1 & 0.5 & 0 & 0.5 \\
$e_{3}$ & 0.9 & 0 & 0 & 0.9 & 0 & 0.9 \\
$e_{4}$ & 0 & 0.7 & 0 & 0 & 0.7 & 0 \\
\hline
\end{tabular}

$\mathscr{A}=\left\{\left(u_{1}, 0.3\right),\left(u_{2}, 0.4\right),\left(u_{3}, 0\right),\left(u_{4}, 0\right),\left(u_{5}, 0\right),\left(u_{6}, 0\right)\right\}$

$\underline{\operatorname{MSFRA}}_{\operatorname{MSFAS}}(\mathscr{A})=\left\{\left(u_{1}, 0\right),\left(u_{2}, 0\right),\left(u_{3}, 0\right),\left(u_{4}, 0\right),\left(u_{5}, 0\right),\left(u_{6}, 0\right)\right\}$

$\overline{\operatorname{MSFRA}}_{M S F A S}(\mathscr{A})=\left\{\left(u_{1}, 0.3\right),\left(u_{2}, 0.7\right),\left(u_{3}, 0\right),\left(u_{4}, 0.2\right),\left(u_{5}, 0.7\right),\left(u_{6}, 0.2\right)\right\}$

$\mathscr{B}=\left\{\left(u_{1}, 0\right),\left(u_{2}, 0.4\right),\left(u_{3}, 0\right),\left(u_{4}, 0.7\right),\left(u_{5}, 0\right),\left(u_{6}, 0\right)\right\}$

$\underline{\operatorname{MSFRA}}_{M S F A S}(\mathscr{B})=\left\{\left(u_{1}, 0\right),\left(u_{2}, 0\right),\left(u_{3}, 0\right),\left(u_{4}, 0\right),\left(u_{5}, 0\right),\left(u_{6}, 0\right)\right\}$

$\overline{\operatorname{MSFRA}}_{\text {MSFAS }}(\mathscr{B})=\left\{\left(u_{1}, 0.2\right),\left(u_{2}, 0.7\right),\left(u_{3}, 0\right),\left(u_{4}, 0.7\right),\left(u_{5}, 0.7\right),\left(u_{6}, 0.2\right)\right\}$

$\overline{\operatorname{MSFRA}}_{M S F A S}(\mathscr{A}) \cup \overline{M S F R A}_{M S F A S}(\mathscr{B})=\left\{\left(u_{1}, 0.3\right),\left(u_{2}, 0.7\right),\left(u_{3}, 0\right),\left(u_{4}, 0.7\right),\left(u_{5}, 0.7\right)\right.$, $\left.\left(u_{6}, 0.2\right)\right\}$

$\mathscr{A} \cup \mathscr{B}=\left\{\left(u_{1}, 0.3\right),\left(u_{2}, 0.4\right),\left(u_{3}, 0\right),\left(u_{4}, 0.7\right),\left(u_{5}, 0\right),\left(u_{6}, 0\right)\right\}$

$\overline{M S F R A}_{M S F A S}(\mathscr{A} \cup \mathscr{B})=\left\{\left(u_{1}, 0.3\right),\left(u_{2}, 0.7\right),\left(u_{3}, 0\right),\left(u_{4}, 0.7\right),\left(u_{5}, 0.7\right),\left(u_{6}, 0.2\right)\right\}$

Note that

$$
\overline{\operatorname{MSFRA}}_{M S F A S}(\mathscr{A}) \cup \overline{M S F R A}_{M S F A S}(\mathscr{B})=\overline{\operatorname{MSFRA}}_{M S F A S}(\mathscr{A} \cup \mathscr{B}) .
$$

$\mathscr{A} \cap \mathscr{B}=\left\{\left(u_{1}, 0\right),\left(u_{2}, 0.4\right),\left(u_{3}, 0\right),\left(u_{4}, 0\right),\left(u_{5}, 0\right),\left(u_{6}, 0\right)\right\}$

$\overline{\operatorname{MSFRA}}_{M S F A S}(\mathscr{A} \cap \mathscr{B})=\left\{\left(u_{1}, 0\right),\left(u_{2}, 0.7\right),\left(u_{3}, 0\right),\left(u_{4}, 0\right),\left(u_{5}, 0.7\right),\left(u_{6}, 0\right)\right\}$

$\underline{M S F R A}_{M S F A S}(\mathscr{A} \cap \mathscr{B})=\left\{\left(u_{1}, 0\right),\left(u_{2}, 0\right),\left(u_{3}, 0\right),\left(u_{4}, 0\right),\left(u_{5}, 0\right),\left(u_{6}, 0\right)\right\}$

$\underline{\operatorname{MSFRA}}_{M S F A S}(\mathscr{B}) \cap \underline{M S F R A}_{M S F A S}(\mathscr{A})=\underline{M S F R A_{M S F A S}}(\mathscr{A} \cap \mathscr{B})$.

$\overline{\operatorname{MSFRA}}_{M S F A S}(\mathscr{A}) \cap \overline{\operatorname{MSFRA}}_{M S F A S}(\mathscr{B})=\left\{\left(u_{1}, 0.2\right),\left(u_{2}, 0.7\right),\left(u_{3}, 0\right),\left(u_{4}, 0.2\right),\left(u_{5}, 0.7\right)\right.$, $\left.\left(u_{6}, 0.2\right)\right\}$

Where

$\overline{\operatorname{MSFRA}}_{M S F A S}(\mathscr{A}) \cap \overline{\operatorname{MSFRA}}_{M S F A S}(\mathscr{B}) \nsubseteq \overline{\operatorname{MSFRA}}_{M S F A S}(\mathscr{A} \cap \mathscr{B})$.

$\mathscr{C}=\left\{\left(u_{1}, 0\right),\left(u_{2}, 0.4\right),\left(u_{3}, 0.6\right),\left(u_{4}, 0\right),\left(u_{5}, 0\right),\left(u_{6}, 0\right)\right\}$

$\underline{\operatorname{MSFRA}}_{M S F A S}(\mathscr{C})=\left\{\left(u_{1}, 0\right),\left(u_{2}, 0\right),\left(u_{3}, 0.6\right),\left(u_{4}, 0\right),\left(u_{5}, 0\right),\left(u_{6}, 0\right)\right\}$ 


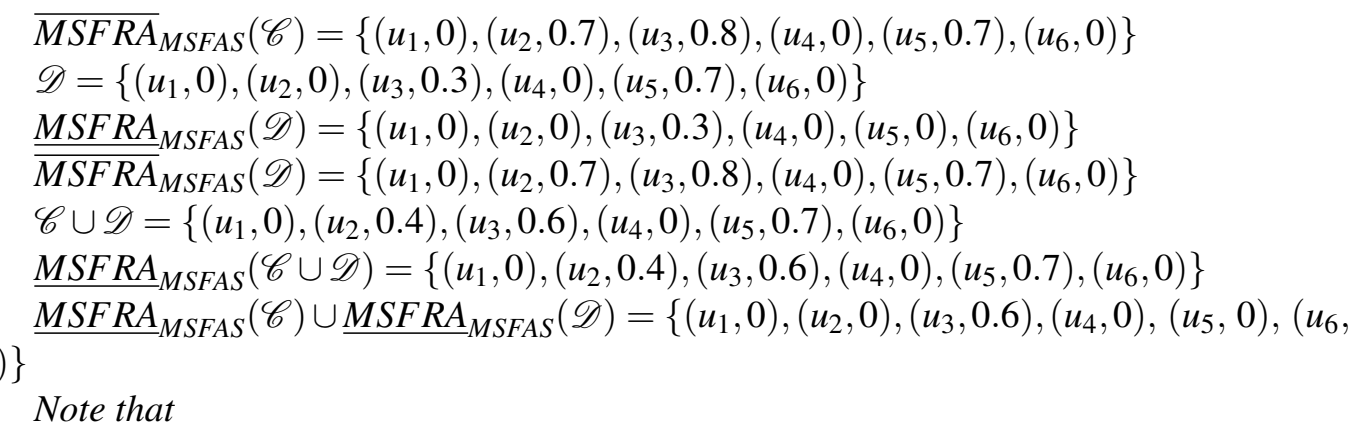

$$
\underline{\operatorname{MSFRA}}_{M S F A S}(\mathscr{C} \cup \mathscr{D}) \nsubseteq \underline{\operatorname{MSFRA}}_{M S F A S}(\mathscr{C}) \cup \underline{\operatorname{MSFRA}}_{M S F A S}(\mathscr{D}) .
$$

$\overline{\operatorname{MSFRA}}_{M S F A S}(\mathscr{C} \cup \mathscr{D})=\left\{\left(u_{1}, 0\right),\left(u_{2}, 0.7\right),\left(u_{3}, 0.8\right),\left(u_{4}, 0\right),\left(u_{5}, 0.7\right),\left(u_{6}, 0\right)\right\}$

$\mathscr{C} \cap \mathscr{D}=\left\{\left(u_{1}, 0\right),\left(u_{2}, 0\right),\left(u_{3}, 0.3\right),\left(u_{4}, 0\right),\left(u_{5}, 0\right),\left(u_{6}, 0\right)\right\}$

$\underline{\operatorname{MSFRA}}_{\text {MSFAS }}(\mathscr{C} \cap \mathscr{D})=\left\{\left(u_{1}, 0\right),\left(u_{2}, 0\right),\left(u_{3}, 0.3\right),\left(u_{4}, 0\right),\left(u_{5}, 0\right),\left(u_{6}, 0\right)\right\}$

Note that

$$
\underline{\operatorname{MSFRA}}_{M S F A S}(\mathscr{C} \cap \mathscr{D})=\underline{\operatorname{MSFRA}}_{M S F A S}(\mathscr{C}) \cap \underline{\operatorname{MSFRA}}_{M S F A S}(\mathscr{D}) .
$$

Remark 3 In general

$$
\begin{aligned}
& \overline{\operatorname{MSFRA}}_{M S F A S}\left(\mathscr{A}^{c}\right) \nsubseteq(\underline{\operatorname{MSFRA}} \underline{M S F A S}(\mathscr{A}))^{c} \\
& \overline{\operatorname{MSFRA}}_{M S F A S}\left(\mathscr{A}^{c}\right) \nsupseteq\left(\underline{\operatorname{MSFRA}}_{M S F A S}(\mathscr{A})\right)^{c}, \\
& \left(\overline{\operatorname{MSFRA}}_{M \operatorname{MSAS}}(\mathscr{A})\right)^{c} \nsubseteq \underline{\operatorname{MSFRA}}_{M S F A S}\left(\mathscr{A}^{c}\right)
\end{aligned}
$$

and

$$
\left(\overline{\operatorname{MSFRA}}_{M S F A S}(\mathscr{A})\right)^{c} \nsupseteq \underline{\operatorname{MSFRA}}_{M S F A S}\left(\mathscr{A}^{c}\right) .
$$

Example 2 Let $U=\left\{u_{1}, u_{2}, u_{3}, u_{4}, u_{5}, u_{6}, u_{7}, u_{8}, u_{9}\right\}$ be the set of nine electricity provider operators (universe set) and $A=\left\{e_{1}, e_{2}, e_{3}, e_{4}\right\} \subseteq E$, where $e_{1}$ represents the online billing facility, $e_{2}$ represents fluctuation in voltage, $e_{3}$ represents life security breakers, $e_{4}$ represents after sale service. The soft fuzzy set $(\tilde{F}, A)$ is representing this data in Table 2.

$$
\begin{aligned}
& \tilde{F}: A \rightarrow P(U) \\
& \varphi: U \rightarrow P(A)
\end{aligned}
$$

Table 2. Soft set $(F, A)$

\begin{tabular}{l|lllllllll}
\hline & $u_{1}$ & $u_{2}$ & $u_{3}$ & $u_{4}$ & $u_{5}$ & $u_{6}$ & $u_{7}$ & $u_{8}$ & $u_{9}$ \\
\hline$e_{1}$ & 0.9 & 0.7 & 0.2 & 0.7 & 0.7 & 0.8 & 0 & 0 & 0 \\
$e_{2}$ & 0.8 & 0 & 0.3 & 0.9 & 0 & 0.9 & 0 & 0 & 0 \\
$e_{3}$ & 1 & 0 & 0 & 0.2 & 0 & 0.6 & 0 & 0 & 0 \\
$e_{4}$ & 0 & 0.3 & 0 & 0 & 0.4 & 0 & 0 & 0 & 0 \\
\hline
\end{tabular}


Then the MSFAS $(U, \varphi)$ will be $\varphi\left(u_{1}\right)=\left\{\left(e_{1}, 0.9\right),\left(e_{2}, 0.8\right),\left(e_{3}, 1\right)\right\}, \varphi\left(u_{2}\right)=\left\{\left(e_{1}, 0.7\right),\left(e_{4}, 0.4\right)\right\}$, $\varphi\left(u_{3}\right)=\left\{\left(e_{1}, 0.2\right),\left(e_{2}, 0.3\right)\right\}, \varphi\left(u_{4}\right)=\left\{\left(e_{1}, 0.7\right),\left(e_{2}, 0.9\right),\left(e_{3}, 0.2\right)\right\}, \varphi\left(u_{5}\right)=\left\{\left(e_{1}, 0.7\right),\left(e_{4}, 0.4\right)\right\}=$ $\varphi\left(u_{2}\right), \varphi\left(u_{6}\right)=\left\{\left(e_{1}, 0.8\right),\left(e_{2}, 0.9\right),\left(e_{3}, 0.6\right)\right\},\left(\varphi\left(u_{6}\right)\right)_{0}=\left(\varphi\left(u_{4}\right)\right)_{0}=\left(\varphi\left(u_{1}\right)\right)_{0}, \varphi\left(u_{7}\right)=$ $\emptyset=\varphi\left(u_{8}\right)=\varphi\left(u_{9}\right)$.

If we take $\mathscr{A}=\left\{\left(u_{1}, 0.3\right),\left(u_{2}, 0.4\right),\left(u_{3}, 1\right),\left(u_{4}, 0\right),\left(u_{5}, 1\right),\left(u_{6}, 0\right),\left(u_{7}, 0.2\right),\left(u_{8}, 0\right),\left(u_{9}\right.\right.$, $1)\}$, then $\mathscr{A}^{c}=\left\{\left(u_{1}, 0.7\right),\left(u_{2}, 0.6\right),\left(u_{3}, 0\right),\left(u_{4}, 1\right),\left(u_{5}, 0\right),\left(u_{6}, 1\right),\left(u_{7}, 0.8\right),\left(u_{8}, 1\right),\left(u_{9}\right.\right.$, $0)\}$.

$(\mathscr{A})_{0}=\left\{u_{1}, u_{2}, u_{3}, u_{5}, u_{7}, u_{9}\right\},\left((\mathscr{A})_{0}\right)^{c}=\left\{u_{4}, u_{6}, u_{8}\right\}$

$\left(\mathscr{A}^{c}\right)_{0}=\left\{u_{1}, u_{2}, u_{4}, u_{6}, u_{7}, u_{8}\right\},\left(\left(\mathscr{A}^{c}\right)_{0}\right)^{c}=\left\{u_{3}, u_{5}, u_{9}\right\}$

Next we calculate some approximations.

$\underline{\operatorname{MSFRA}}_{M S F A S}(\mathscr{A})=\left\{\left(u_{1}, 0.3\right),\left(u_{2}, 0.4\right),\left(u_{3}, 0.3\right),\left(u_{4}, 0\right),\left(u_{5}, 0.7\right),\left(u_{6}, 0\right),\left(u_{7}, 0\right)\right.$, $\left.\left(u_{8}, 0\right),\left(u_{9}, 0\right)\right\}$

$\left(\underline{\operatorname{MSFRA}}_{M S F A S}(\mathscr{A})\right)^{c}=\left\{\left(u_{1}, 0.7\right),\left(u_{2}, 0.6\right),\left(u_{3}, 0.7\right),\left(u_{4}, 1\right),\left(u_{5}, 0.3\right),\left(u_{6}, 1\right),\left(u_{7}, 1\right)\right.$, $\left.\left(u_{8}, 1\right),\left(u_{9}, 1\right)\right\}$

$\operatorname{MSFRA}_{M S F A S}\left(\mathscr{A}^{c}\right)=\left\{\left(u_{1}, 0.7\right),\left(u_{2}, 0\right),\left(u_{3}, 0\right),\left(u_{4}, 0.9\right),\left(u_{5}, 0\right),\left(u_{6}, 0.9\right),\left(u_{7}, 0\right),\left(u_{8}, 0\right)\right.$, $\left.\left(u_{9}, 0\right)\right\}$

$\left(\underline{M S F R A}_{M S F A S}\left(\mathscr{A}^{c}\right)\right)^{c}=\left\{\left(u_{1}, 0.3\right),\left(u_{2}, 1\right),\left(u_{3}, 1\right),\left(u_{4}, 0.1\right),\left(u_{5}, 1\right),\left(u_{6}, 0.1\right),\left(u_{7}, 1\right)\right.$, $\left.\left(u_{8}, 1\right),\left(u_{9}, 1\right)\right\}$

$\overline{\operatorname{MSFRA}}_{M S F A S}(\mathscr{A})=\left\{\left(u_{1}, 0.8\right),\left(u_{2}, 0.4\right),\left(u_{3}, 1\right),\left(u_{4}, 0\right),\left(u_{5}, 1\right),\left(u_{6}, 0\right),\left(u_{7}, 0.2\right),\left(u_{8}, 0\right)\right.$, $\left.\left(u_{9}, 1\right)\right\}$

$\left(\overline{\operatorname{MSFRA}}_{M S F A S}(\mathscr{A})\right)^{c}=\left\{\left(u_{1}, 0.2\right),\left(u_{2}, 0.6\right),\left(u_{3}, 0\right),\left(u_{4}, 1\right),\left(u_{5}, 0\right),\left(u_{6}, 1\right),\left(u_{7}, 0.8\right)\right.$, $\left.\left(u_{8}, 1\right),\left(u_{9}, 0\right)\right\}$

$\overline{\operatorname{MSFRA}}_{M S F A S}\left(\mathscr{A}^{c}\right)=\left\{\left(u_{1}, 0.8\right),\left(u_{2}, 0.6\right),\left(u_{3}, 0\right),\left(u_{4}, 1\right),\left(u_{5}, 0.4\right),\left(u_{6}, 1\right),\left(u_{7}, 0.8\right)\right.$, $\left.\left(u_{8}, 1\right),\left(u_{9}, 0\right)\right\}$

$\left(\overline{\operatorname{MSFRA}}_{M S F A S}\left(\mathscr{A}^{c}\right)\right)^{c}=\left\{\left(u_{1}, 0.2\right),\left(u_{2}, 0.4\right),\left(u_{3}, 1\right),\left(u_{4}, 0\right),\left(u_{5}, 0.6\right),\left(u_{6}, 0\right),\left(u_{7}, 0.2\right)\right.$, $\left.\left(u_{8}, 0\right),\left(u_{9}, 1\right)\right\}$

Note that

$$
\left(\underline{\operatorname{MSFRA}}_{M S F A S}(\mathscr{A})\right)^{c}\left(u_{1}\right)<\overline{\operatorname{MSFRA}}_{M S F A S}\left(\mathscr{A}^{c}\right)\left(u_{1}\right)
$$

and

$$
\overline{\operatorname{MSFRA}}_{M S F A S}\left(\mathscr{A}^{c}\right)\left(u_{3}\right)<\left(\underline{\operatorname{MSFRA}}_{M S F A S}(\mathscr{A})\right)^{c}\left(u_{3}\right) .
$$

Thus

$$
\overline{\operatorname{MSFRA}}_{M S F A S}\left(\mathscr{A}^{c}\right) \nsubseteq\left(\underline{\operatorname{MSFRA}}_{M S F A S}(\mathscr{A})\right)^{c}
$$

and

$$
\overline{\operatorname{MSFRA}}_{M S F A S}\left(\mathscr{A}^{c}\right) \nsupseteq\left(\underline{\operatorname{MSFRA}}_{M S F A S}(\mathscr{A})\right)^{c} .
$$

Note that

$$
\underline{\operatorname{MSFRA}}_{M S F A S}\left(\mathscr{A}^{c}\right)\left(u_{1}\right)>\left(\overline{\operatorname{MSFRA}}_{M S F A S}(\mathscr{A})\right)^{c}\left(u_{1}\right)
$$

and

$$
\left(\overline{\operatorname{MSFRA}}_{M S F A S}(\mathscr{A})\right)^{c}\left(u_{2}\right)>\underline{\operatorname{MSFRA}}_{M S F A S}\left(\mathscr{A}^{c}\right)\left(u_{2}\right) .
$$


So

$$
\left(\overline{\operatorname{MSFRA}}_{M S F A S}(\mathscr{A})\right)^{c} \nsubseteq \underline{\operatorname{MSFRA}} \underline{M S F A S}\left(\mathscr{A}^{c}\right)
$$

and

$$
\left(\overline{\operatorname{MSFRA}}_{M S F A S}(\mathscr{A})\right)^{c} \nsupseteq \underline{\operatorname{MSFRA}}_{M S F A S}\left(\mathscr{A}^{c}\right) .
$$

It is easy to note that

$$
\left(\overline{\operatorname{MSFRA}}_{M S F A S}\left(\mathscr{A}^{c}\right)\right)^{c} \nsubseteq \underline{\operatorname{MSFRA}} \underline{M S F A S}_{(\mathscr{A})}
$$

and

$$
\left(\overline{\operatorname{MSFRA}}_{M S F A S}\left(\mathscr{A}^{c}\right)\right)^{c} \nsupseteq \underline{\operatorname{MSFRA}}_{M S F A S}(\mathscr{A}) .
$$

Therefore

$$
\overline{\operatorname{MSFRA}}_{M S F A S}(\mathscr{A}) \nsubseteq\left(\underline{\operatorname{MSFRA}}_{M S F A S}\left(\mathscr{A}^{c}\right)\right)^{c}
$$

and

$$
\overline{\operatorname{MSFRA}}_{M S F A S}(\mathscr{A}) \nsupseteq\left(\underline{\operatorname{MSFRA}}_{M S F A S}\left(\mathscr{A}^{c}\right)\right)^{c} .
$$

$\underline{\operatorname{MSFRA}}_{M S F A S}(\mathscr{A})=\left\{\left(u_{1}, 0.3\right),\left(u_{2}, 0.4\right),\left(u_{3}, 0.3\right),\left(u_{4}, 0\right),\left(u_{5}, 0.7\right),\left(u_{6}, 0\right),\left(u_{7}, 0\right)\right.$, $\left.\left(u_{8}, 0\right),\left(u_{9}, 0\right)\right\}$

$\left(\underline{\operatorname{MSFRA}}_{M S F A S}(\mathscr{A})\right)_{0}=\left\{u_{1}, u_{2}, u_{3}, u_{5}\right\},\left(\left(\underline{\operatorname{MSFRA}}_{M S F A S}(\mathscr{A})\right)_{0}\right)^{c}=\left\{u_{4}, u_{6}, u_{7}, u_{8}, u_{9}\right\}$ $\underline{\operatorname{MSFRA}}_{M S F A S}\left(\underline{\operatorname{MSFRA}}_{M S F A S}(\mathscr{A})\right)=\left\{\left(u_{1}, 0.3\right),\left(u_{2}, 0.4\right),\left(u_{3}, 0.3\right),\left(u_{4}, 0\right),\left(u_{5}, 0.7\right)\right.$, $\left.\left(u_{6}, 0\right),\left(u_{7}, 0\right),\left(u_{8}, 0\right),\left(u_{9}, 0\right)\right\}$

$\overline{\operatorname{MSFRA}}_{M S F A S}\left(\underline{\operatorname{MSFRA}}_{M S F A S}(\mathscr{A})\right)=\left\{\left(u_{1}, 0.8\right),\left(u_{2}, 0.4\right),\left(u_{3}, 0.3\right),\left(u_{4}, 0\right),\left(u_{5}, 0.7\right)\right.$, $\left.\left(u_{6}, 0\right),\left(u_{7}, 0\right),\left(u_{8}, 0\right),\left(u_{9}, 0\right)\right\}$

Note that

$$
\underline{\operatorname{MSFRA}}_{M S F A S}\left(\underline{\operatorname{MSFRA}}_{M S F A S}(\mathscr{A})\right)=\underline{\operatorname{MSFRA}}_{M S F A S}(\mathscr{A})
$$

and

$$
\overline{\operatorname{MSFRA}}_{M S F A S}\left(\underline{\operatorname{MSFRA}}_{M S F A S}(\mathscr{A})\right) \supset \underline{\operatorname{MSFRA}}_{M S F A S}(\mathscr{A}) .
$$

$\overline{\operatorname{MSFRA}}_{M S F A S}(\mathscr{A})=\left\{\left(u_{1}, 0.8\right),\left(u_{2}, 0.4\right),\left(u_{3}, 1\right),\left(u_{4}, 0\right),\left(u_{5}, 1\right),\left(u_{6}, 0\right),\left(u_{7}, 0.2\right),\left(u_{8}, 0\right)\right.$, $\left.\left(u_{9}, 1\right)\right\}$

$\overline{\operatorname{MSFRA}}_{M S F A S}(\mathscr{A})=\left\{u_{1}, u_{2}, u_{3}, u_{5}, u_{7}, u_{9}\right\},\left(\overline{\operatorname{MSFRA}}_{M S F A S}(\mathscr{A})\right)^{c}=\left\{u_{4}, u_{6}, u_{8}\right\}$ $\underline{\operatorname{MSFRA}}_{M S F A S}\left(\overline{\operatorname{MSFRA}}_{M S F A S}(\mathscr{A})\right)=\left\{\left(u_{1}, 0.8\right),\left(u_{2}, 0.4\right),\left(u_{3}, 0.3\right),\left(u_{4}, 0\right),\left(u_{5}, 0.7\right)\right.$, $\left.\left(u_{6}, 0\right),\left(u_{7}, 0\right),\left(u_{8}, 0\right),\left(u_{9}, 0\right)\right\}$

$\overline{\operatorname{MSFRA}}_{M S F A S}\left(\overline{\operatorname{MSFRA}}_{M S F A S}(\mathscr{A})\right)=\left\{\left(u_{1}, 0.8\right),\left(u_{2}, 0.4\right),\left(u_{3}, 1\right),\left(u_{4}, 0\right),\left(u_{5}, 1\right),\left(u_{6}, 0\right)\right.$, $\left.\left(u_{7}, 0.2\right),\left(u_{8}, 0\right),\left(u_{9}, 1\right)\right\}$

Thus

$$
\underline{\operatorname{MSFRA}}_{M S F A S}\left(\overline{\operatorname{MSFRA}}_{M S F A S}(\mathscr{A})\right) \subset \overline{\operatorname{MSFRA}}_{M S F A S}(\mathscr{A})
$$

and

$$
\overline{\operatorname{MSFRA}}_{M S F A S}\left(\overline{\operatorname{MSFRA}}_{M S F A S}(\mathscr{A})\right)=\overline{\operatorname{MSFRA}}_{M S F A S}(\mathscr{A}) .
$$




\section{Conclusion}

Fuzzy set theory has been used successfully to handle uncertainty and vagueness in information. Soft rough set theory is applied for the approximation of undefinable sets. But there are several deficiencies while doing approximations of undefinable fuzzy sets not satisfying the basic properties of approximation measure in soft fuzzy approximation space. MSFRSs have been introduced to overcome these deficiencies. MSFRS give better approximations of undefinable fuzzy sets. Our study has potential for further new research directions.

Conflicts of Interest; The authors declare that there is no conflicts of interest regarding the publication of this manuscript.

Acknowledgement; The authors sincerely thank the learned referees for the careful reading and thoughtful comments. The present version of the paper owes much to their precise and kind remarks.

\section{References}

[1] M. I. Ali, F. Feng, X. Liu, W. K. Min And M. Shabir, On some new operations in soft set theory, Computers and Mathematics with Applications 57 (2009) 1547-1553.

[2] I. BEG AND T. RASHID, TOPSIS FOR HESITANT FUZZY LINGUISTIC TERM SETS, International Journal of Intelligent Systems, 28 (2013), 1162-1171.

[3] R. E. Bellman AND L. A. ZAdeh, Decision making in a fuzzy environment, Management Science, 17(4) (1970) 141-164.

[4] D. G. Chen, E. C. C. Tsang, D. S. Yeung And X. Z. WAng, The paremeterization reduction of soft sets and its applications, Computers and Mathematics with Applications 49 (2005) 757-763.

[5] F. Feng, X. LiU, V. Leoreanu-Fotea And Y. B. Jun, Soft sets and soft rough sets, Information Sciences, 181 (2011) 1125-1137.

[6] X. GE, Z. LI AND Y. GE, Topological spaces and soft sets, Journal of Computational Analysis and Applications, 13 (2011) 881-885.

[7] S. GReCO, B. MATARAZZO AND R. SLOWINSKI, Rough set theory for multicriteria decision analysis, European Journal of Operational Research, 129 (2001) 1-47.

[8] P. Hásek, Metamathematics of Fuzzy Logic, Trends in Logic, Springer Netherlands, (1998).

[9] T. Herawan AND M. M. Deris, A soft set approach for association rules mining, Knowledge-Based Systems, 24 (2011) 186-195.

[10] T. B. IwINSKI, Algebraic approach to rough sets, Bulletin of the Polish Academy of Sciences, Mathematics, 35 (9-10) (1987).

[11] Y. B. Jun, Roughness of ideals in BCK-algebra, Scientiae Mathematicae Japonica, 57 (1) (2003) 165-169. 
[12] S. J. Kalayathankal And G. S. Singh, A fuzzy soft flood alarm model, Mathematics and Computers in Simulation, 80 (2010) 887-893.

[13] Z. Kong, L. Q. GAO, L. F. WANG AND S. LI, The normal parameter reduction of soft sets and its algorithm, Computers and Mathematics with Applications 56 (2008) 3029-3037.

[14] P. K. MAJi, R. BISWAS AND A. R. RoY, Fuzzy soft sets, The Journal of Fuzzy Mathematics 9 (2001) 589-602.

[15] P. K. MAJI, A. R. ROY AND R. BISWAS, An application of soft sets in a decision making problem, Computers and Mathematics with Applications 44 (2002) 1077-1083.

[16] P. K. MAJi, R. Biswas And R. Roy, Soft set theory, Computers and Mathematics with Applications, 45 (2003) 555-562.

[17] D. Meng, X. ZHAng AND K. QIN, Soft rough fuzzy sets and soft fuzzy rough sets, Computers and Mathematics with Applications, 62 (2011) 4635-4645.

[18] D. Molodtsov, Soft set theory - first results, Computers and Mathematics with Applications, 37 (1999) 19-31.

[19] Z. PAWLAK, Rough sets, International Journal of Computing and Information Sciences, 11 (1982) 341-356.

[20] K. Y. QIN AND Z. Y. Hong, On soft equality, Journal of Computational and Applied Mathematics 234 (2010) 1347-1355.

[21] A. R. RoY AND P. K. MAJI, A fuzzy soft set theoretic approach to decision making problems, Journal of Computational and Applied Mathematics 203 (2007) 412-418.

[22] Z. XIAO, K. GONG AND Y. ZOU, A combined forecasting approach based on fuzzy soft sets, Journal of Computational and Applied Mathematics 228 (2009) 326-333.

[23] L. A. ZADEH, Fuzzy sets, Information and Control, 8 (1965) 338-353.

[24] H. J. Zimmermann, Fuzzy Set Theory and its Applications, second edition, Kluwer Academic Publishers, Boston, (1991). 